\title{
Carbon Dots Decorated Carbon Nitride Nanoparticles for Enhanced Photodynamic Therapy against Hypoxic Tumor via Water Splitting
}

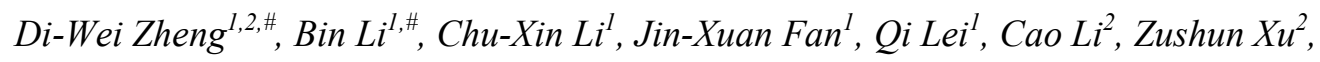
Xian-Zheng Zhang ${ }^{1, *}$

${ }^{1}$ Key Laboratory of Biomedical Polymers of Ministry of Education, Institute for Advanced Studies (IAS), Department of Chemistry, Wuhan University, Wuhan 430072, P. R. China.

${ }^{2}$ Hubei Collaborative Innovation Center for Advanced Organic Chemical Materials, Key Laboratory for the Green Preparation and Application of Functional Materials of Ministry of Education, Hubei University, Wuhan, Hubei 430062, P. R. China

\footnotetext{
* Corresponding author. Email address: xz-zhang@whu.edu.cn

\# These authors contributed equally to this work.
} 


\section{METHODS}

Materials. Citric acid, urea, N, N-dimethyl formamide (DMF), piperidine, sodium hydrosulfite, triisopropylsilane (TIS) and diisopropylethylamine (DIEA) were purchased from Shanghai Reagent Chemical Co. Fmoc- $\mathrm{NH}_{2}-\mathrm{PEG}_{8}-\mathrm{COOH}$ was purchased from Zhoubei Technology Co. Ltd. (Hangzhou, China). Trifluoroacetic acid (TFA) and protoporphyrin IX (PpIX) were purchased from Aladdin Industrial Corporation. N-(9-Fluorenylmethoxycarbonyl) (Fmoc) protected amino acids Fmoc-Arg(Pbf)-OH, Fmoc-Gly-OH and Fmoc-Asp(OtBu)-OH, o-Benzotriazol-1yl-N,N,N',N'-tetramethyluronium hexafluorophosphate (HBTU) and 1-Hydroxybenzotriazole (HOBt) were purchased from GL biochem (Shanghai) Ltd. 2',7'-Dichlorofluorescin diacetate (DCFH-DA) was purchased from Beyotime Ltd. ROS-ID $^{\text {TM }}$ hypoxia/oxidative stress detection kit was purchased from Enzo Life Sciences Inc. CD31, CA9 and HIF- $\alpha$ antibodies were purchased from Abcam Inc.

Characterization. Transmission electron microscopy (TEM) images were obtained on a JEM-2100 (JEOL) transmission electron microscope. FT-IR spectrum was recorded on a Spectrum Two FT-IR Spectrophotometer (Perkin-Elmer). Zeta potential and particle size were measured by a zeta sizer (Nano ZS, Malvern Instruments). Powder X-ray diffraction (XRD) analysis was performed by using a Rigaku MiniFlex 600 X-ray diffractometer with $\mathrm{Cu}-\mathrm{K} \alpha(\lambda=1.5418 \AA)$. Thermogravimetric analysis (TGA) was performed on a Pyris1 thermogravimetric analyser (Perkin-Elmer) under a nitrogen flow at a heating rate of $10{ }^{\circ} \mathrm{C} \min ^{-1}$. Fourier transform-infrared spectroscopy (FT-IR) of samples were performed by using an Avatar 360 FT-IR spectrophotometer (PerkinElmer). Confocal laser scanning microscope (CLSM) images were obtained on a C1-Si (Nikon) confocal laser scanning microscope. Small animals fluorescence imaging was performed 
with a living image IVIS ${ }^{\circledR}$ spectrum (Perkin-Elmer). CT scanning was performed in Quantum FX microCT (Perkin-Elmer).

$\mathrm{O}_{2}$ Generation of $\mathbf{C C N}$. Herein, sodium hydrosulfite was used to produce deoxygenated PBS. Then, $50 \mathrm{mg}$ of $\mathrm{CCN}$ or $\mathrm{C}_{3} \mathrm{~N}_{4}$ was dispersed in $25 \mathrm{~mL}$ of deoxygenated PBS. After that, $10 \mathrm{~mL}$ of liquid paraffin was added to isolate deoxygenated PBS and air. The solution was illuminated under a laser light $(630 \mathrm{~nm}$ He-Ne laser, $200 \mathrm{~mW} \mathrm{~cm}^{-2}$ ). A DOG-3082 oxygen dissolving meter was used to measure the $\mathrm{O}_{2}$ generation in real-time.

ROS Generation of PCCN. First, DCFH-DA was converted to DCFH according to the previous report. ${ }^{\mathrm{S} 1}$ Briefly, $0.5 \mathrm{~mL}$ of $1 \mathrm{mM}$ DCFH-DA in ethanol was added to $2 \mathrm{~mL}$ of $0.01 \mathrm{~N} \mathrm{NaOH}$. Then, the solution was stirred at room temperature for 30 min. The hydrolysate was then neutralized with $10 \mathrm{~mL}$ of PBS at $\mathrm{pH} 7.4$, and stored on ice until use. Then, $60 \mu \mathrm{L}$ of DCFH solution was added in $3 \mathrm{~mL}$ of PpIX or PCCN (with a PpIX concentration of $10 \mathrm{mg} / \mathrm{L}$ ) contained deoxygenated PBS or normal PBS. The solution was illuminated under a laser light (630 nm He-Ne laser, $40 \mathrm{~mW} \mathrm{~cm}^{-2}$ ). Emission spectra of various solutions were recorded with a fluorescence spectrophotometer.

In Vivo and ex Vivo Fluorescence Imaging. The fluorescence imaging was performed on 4T1 tumor bearing mice. $200 \mu \mathrm{L}$ of PCCN (with a PpIX concentration of $2.5 \mathrm{mg} / \mathrm{kg}$ ) was i.v. injected into mice. Then, mice were imaged at different time point (Pre, 1, 2, 3, 4, 8, 12, 24, 36 and 48 h) with an excitation wavelength of $620 \mathrm{~nm}$ and emission wavelength of $680 \mathrm{~nm}$. For the 3D fluorescence image, transillumin fluorescent model was performed with a living image IVIS ${ }^{\circledR}$ spectrum (Perkin-Elmer) with an excitation wavelength of $620 \mathrm{~nm}$ and emission wavelength of $680 \mathrm{~nm}$. Then, the fluorescence signal was under a 3D 
reconsitution program. CT scanning was performed to obtain structural informations of mice with a Quantum FX microCT (Perkin-Elmer). 3D CT mice structural informations and $3 \mathrm{D}$ fluorescence signal were overlayed in IVIS $\mathbb{}$ spectrum software.

Western Blot Assay. 4T1 cells and MCF-7 cells were lysed and collected. Then, lysate was treated with $50 \mu \mathrm{L}$ of RIPA buffer and resuspended in $50 \mu \mathrm{L}$ of SDS buffer with $1 \% \beta$-mercaptoethanol. Then the samples were heated for 5 min and separated on a $10 \%$ SDS-PAGE (15 $\mu \mathrm{L}$ per lane). After electrophoresis, proteins were transfered to a PVDF membrane (Millipore). The PVDF membranes were then blocked in PBS with 5\% skim milk for $1 \mathrm{~h}$. Intracellular $\alpha v \beta 3$ (Bioss) was detected. The membranes were incubated with the $\alpha v \beta 3$ antibody (Bioss) rabbit anti-mice antibody (1:2000 dilution) overnight at $4{ }^{\circ} \mathrm{C}$ and then with the secondary antibody HRP labeled goat antirabbit IgG (1:3000 dilution, KPL) for 1 h. $\alpha v \beta 3$ was

monitored by enhanced chemiluminescence. GADPH (Abcam, Rabbit) was employed as protein loading control.

\section{References}

S1. Yuan, Y.; Liu, J.; Liu, B. Conjugated-Polyelectrolyte-Based Polyprodrug: Targeted and Image-Guided Photodynamic and Chemotherapy with On-Demand Drug Release upon Irradiation with a Single Light Source. Angew. Chem. Int. Ed. 2014, 53, 7163-7168. 

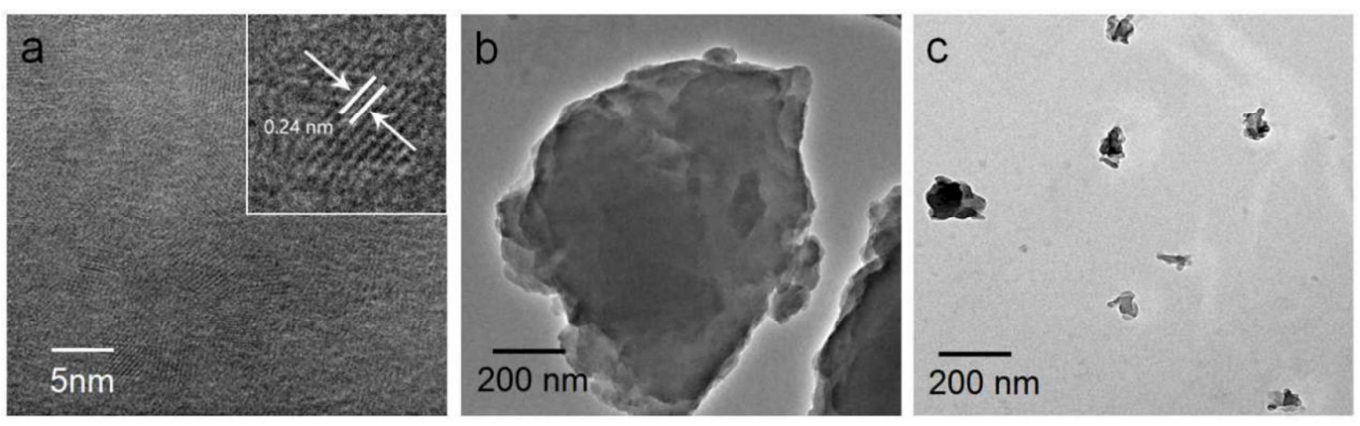

Figure S1. TEM images of (a) carbon dots; (b) CCN composite and (c) PCCN nanoparticles produced by a ball-milling process. 


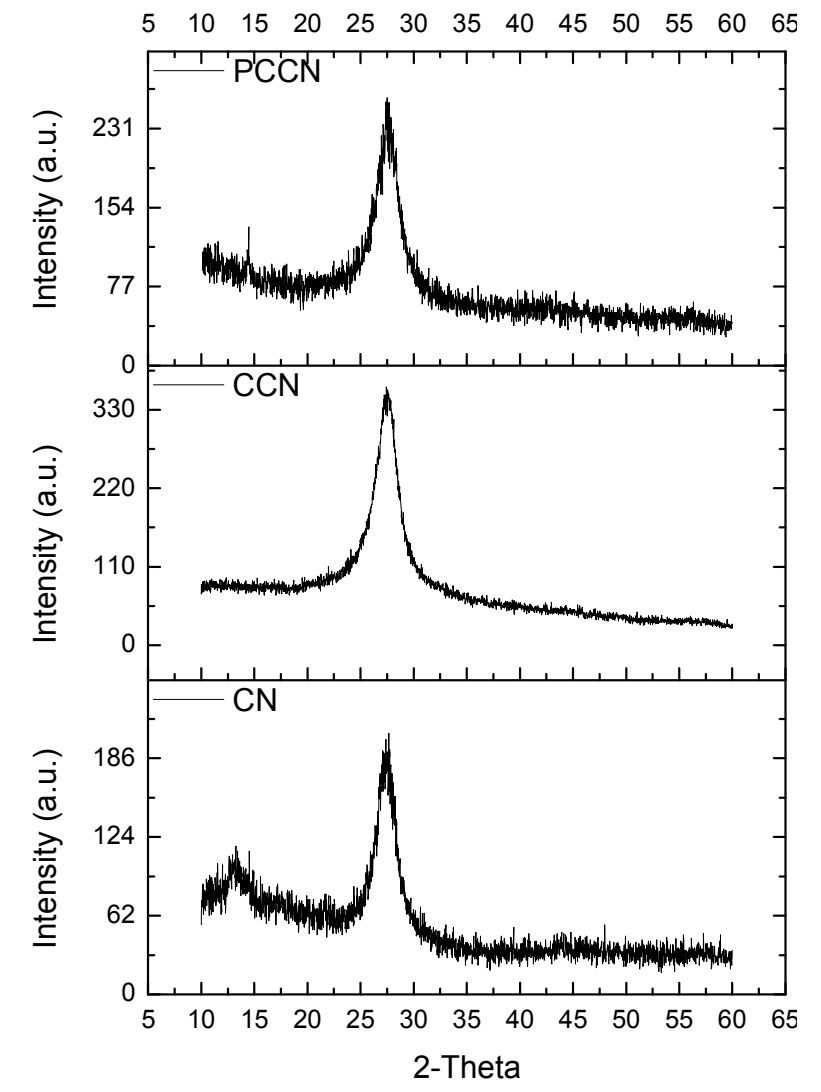

Figure S2. XRD spectrum of PCCN, $\mathrm{CCN}$ and $\mathrm{C}_{3} \mathrm{~N}_{4}$. 

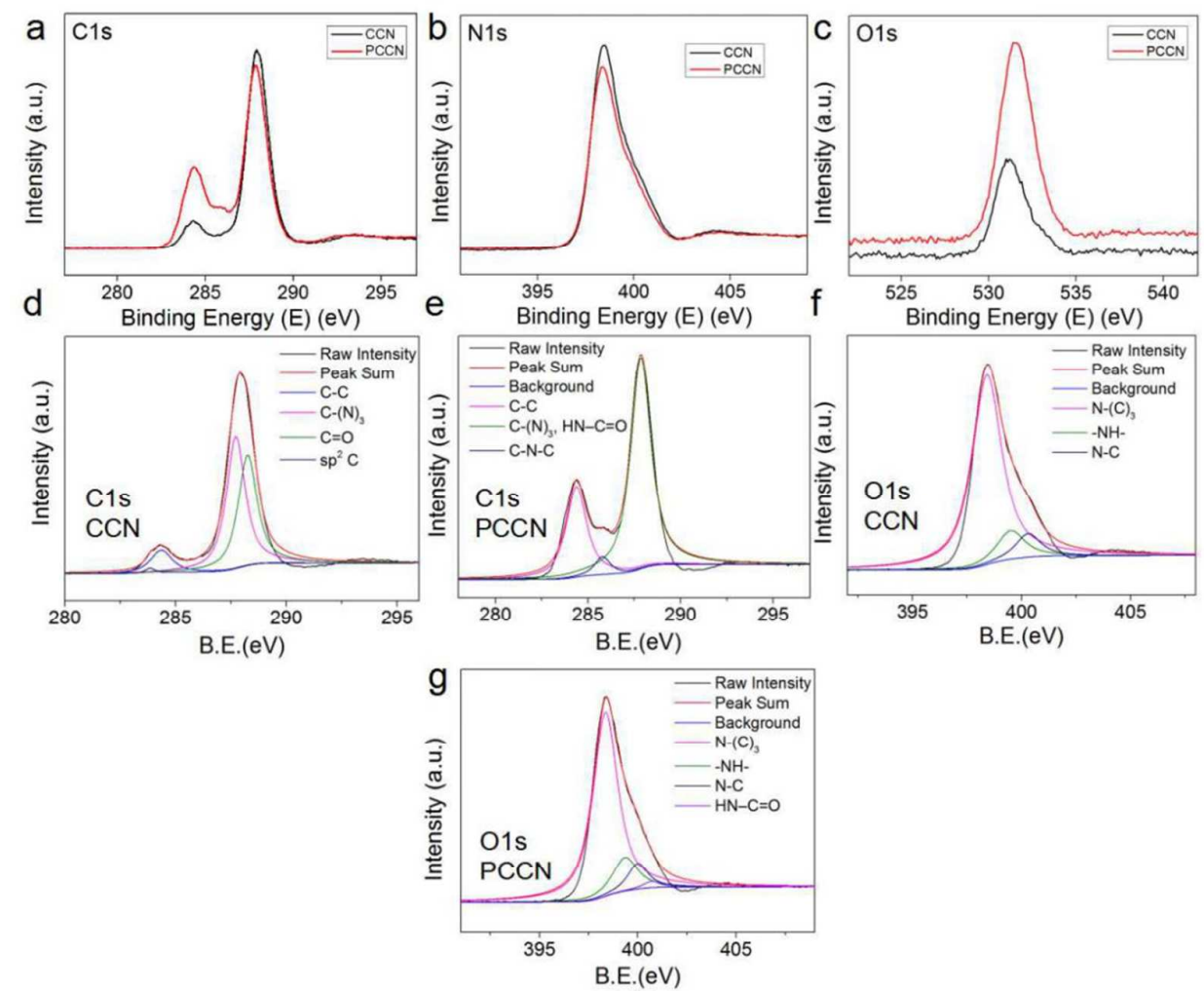

Figure S3. (a) XPS spectrum for C1s orbital of PCCN and CCN; (b) XPS spectrum for N1s orbital of PCCN and CCN; (c) XPS spectrum for O1s orbital of PCCN and CCN (d) XPS de-convoluted spectra for the C1s orbitals of CCN, in which 283.8, 284.4, 287.7 and $288.3 \mathrm{eV}$ represent $\mathrm{sp}^{2} \mathrm{C}, \mathrm{C}-\mathrm{C}, \mathrm{C}-(\mathrm{N})_{3}$ and $\mathrm{C}=\mathrm{O}$, respectively; (e) XPS de-convoluted spectra for the C1s orbitals of PCCN, in which 284.4, 285.8 and $287.9 \mathrm{eV}$ represent $\mathrm{C}-\mathrm{C}, \mathrm{C}-\mathrm{N}-\mathrm{C}$ and $\mathrm{C}-(\mathrm{N}) 3$ together with $\mathrm{HN}-\mathrm{C}=\mathrm{O}$, respectively; (f) XPS de-convoluted spectra for the N1s orbitals of CCN , in which 398.4, 399.5, and $400.3 \mathrm{eV}$ represent $\mathrm{N}-(\mathrm{C})_{3},-\mathrm{NH}-$, and $\mathrm{N}-\mathrm{C}$, respectively; (g) XPS de-convoluted spectra for the N1s orbitals of PCCN , in which 398.4, 399.4, 400.0 and $400.7 \mathrm{eV}$ represent $\mathrm{N}-(\mathrm{C})_{3},-\mathrm{NH}-, \mathrm{N}-\mathrm{C}$ and $\mathrm{HN}-\mathrm{C}=\mathrm{O}$, respectively. 


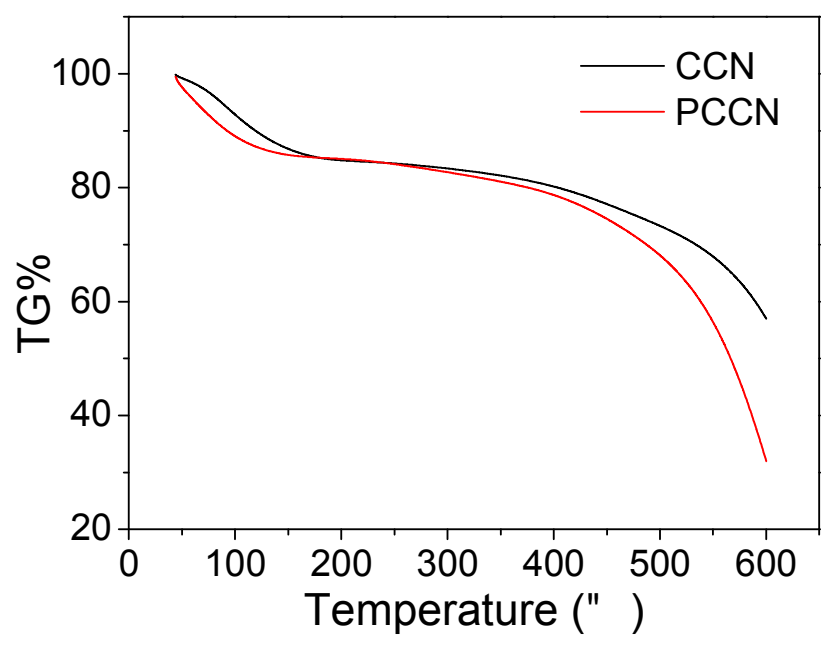

Figure S4. Thermogravimetric analysis of $\mathrm{CCN}$ and PCCN. 


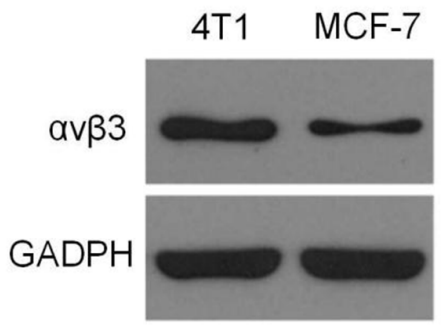

Figure S5. Western blot assay of $\alpha \mathrm{v} \beta 3$ level in 4T1 cells and MCF-7 cells. 

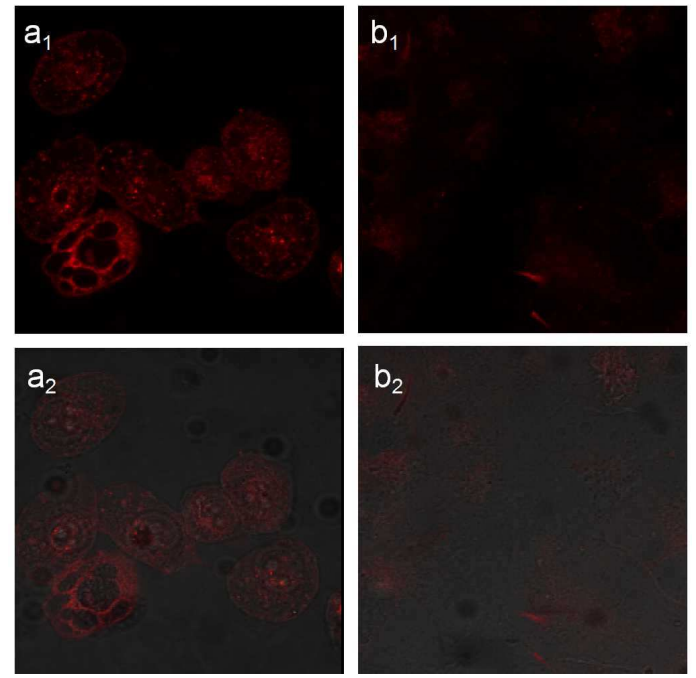

Figure S6. CLSM images of (a) 4T1 cells and (b) MCF-7 cells after $4 \mathrm{~h}$ of PCCN treatment (1: Red fluorescence; 2: Merged). 


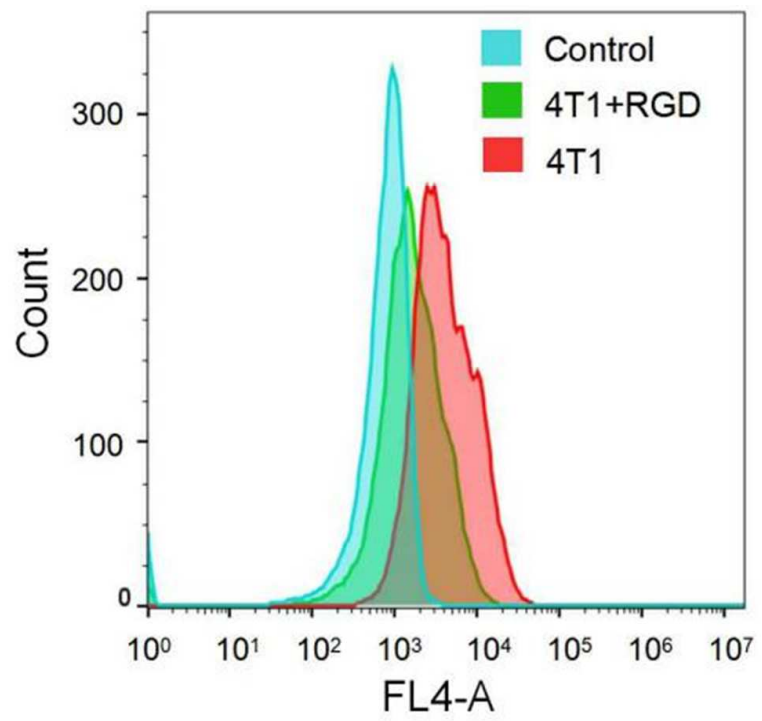

Figure S7. Flow cytometry analysis of $4 \mathrm{~T} 1$ cells treated with $\mathrm{PCCN}$ in the presence and in the absence of free RGD. 


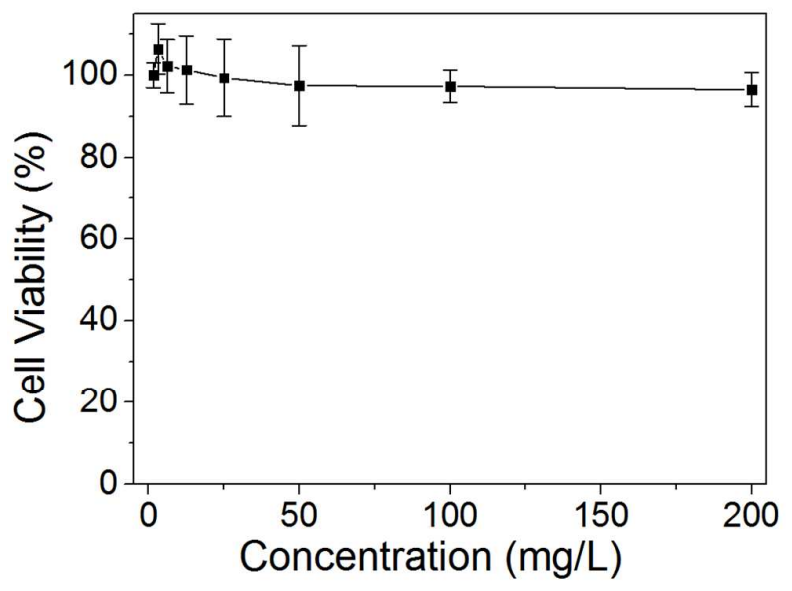

Figure S8. Cell viability assay of PCCN in $4 \mathrm{~T} 1$ cells without laser irradiation. 


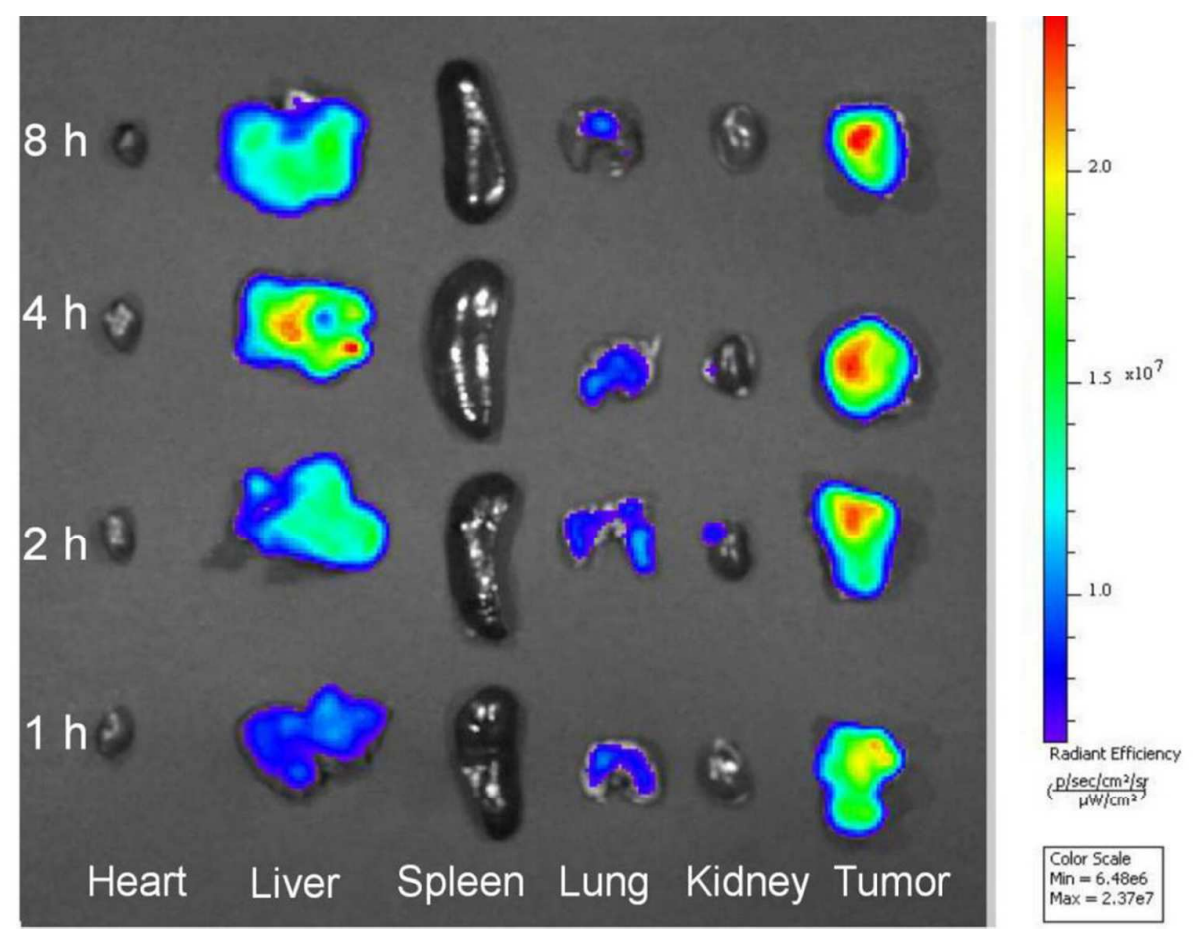

Figure S9. Ex vivo fluorescence imaging of heart, liver, spleen, lung, kidney and tumor $1 \mathrm{~h}, 2 \mathrm{~h}, 4 \mathrm{~h}$ and $8 \mathrm{~h}$ after PCCN treatment. 


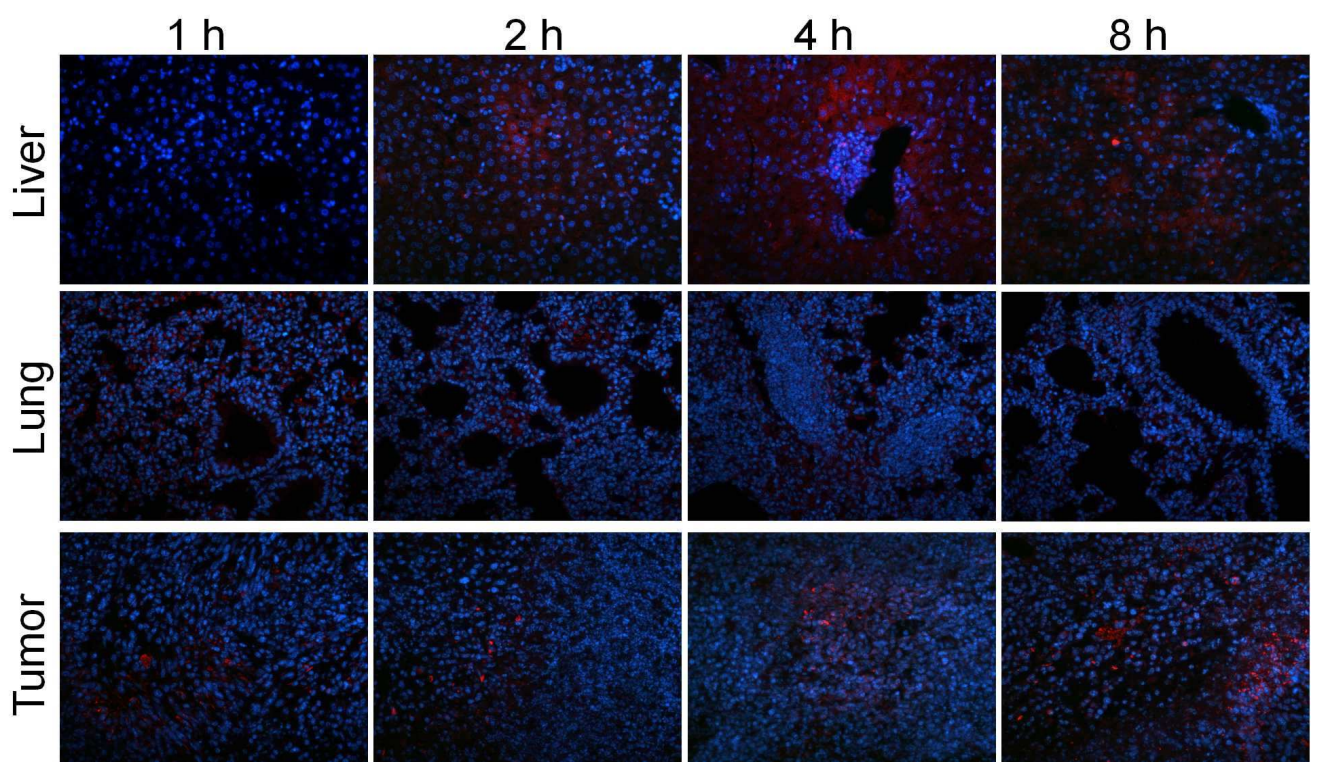

Figure S10. Ex vivo CLSM imaging of liver, lung and tumor $1 \mathrm{~h}, 2 \mathrm{~h}, 4 \mathrm{~h}$ and $8 \mathrm{~h}$ after PCCN treatment. 


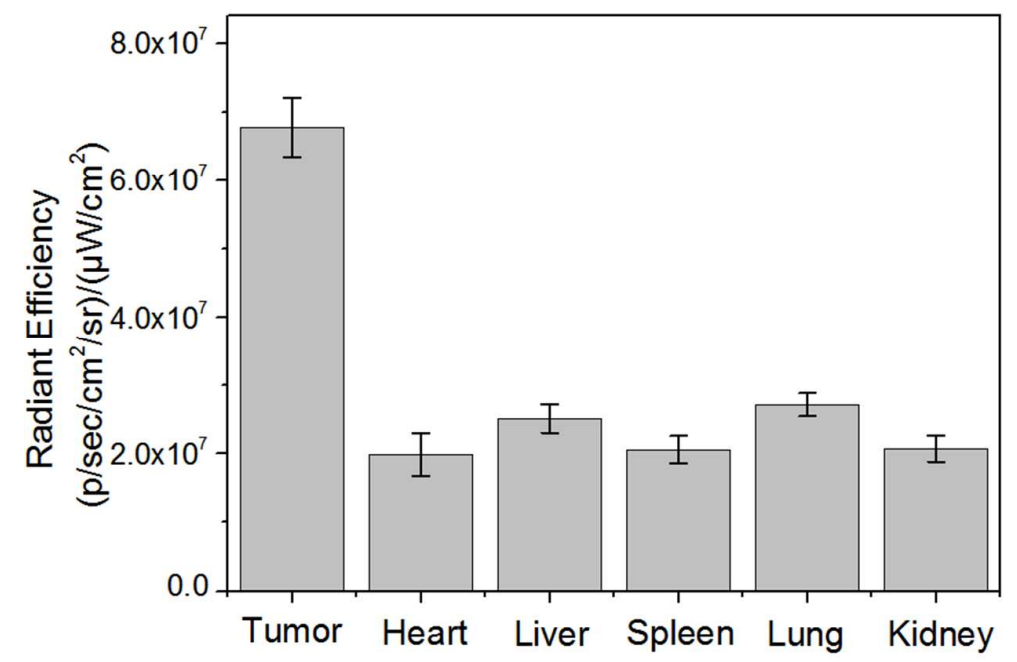

Figure S11. Quantitative analysis of PCCN fluorescence in tumor, liver, heart, lung and kidney $48 \mathrm{~h}$ post-injection. 


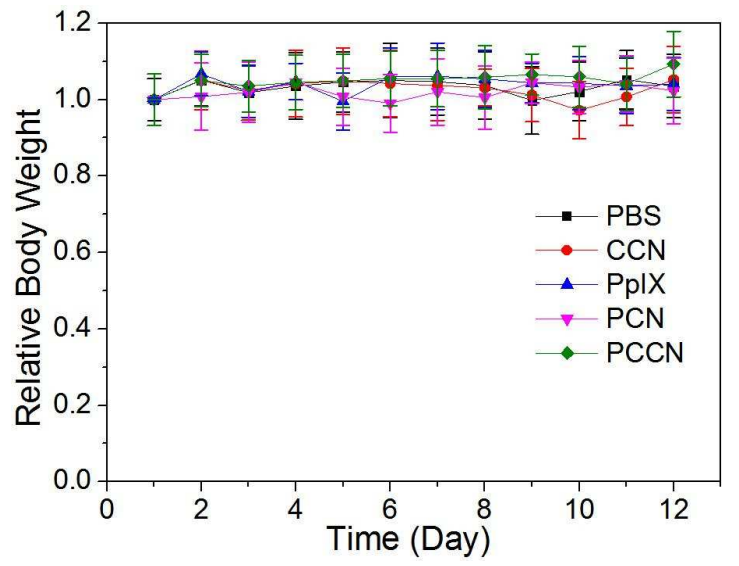

Figure S12. Relative body weight of $\mathrm{PCCN}, \mathrm{PCN}$. $\mathrm{CCN}$ and $\mathrm{C}_{3} \mathrm{~N}_{4}$ treated mice. 


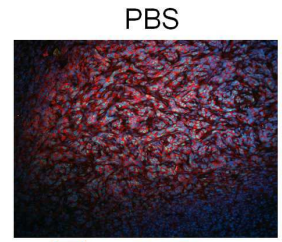

PpIX

PCN
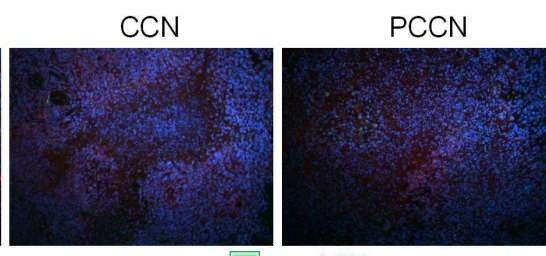

DAPI

回
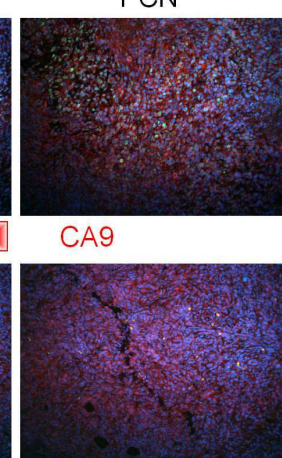

CA9

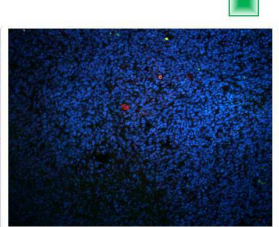

$H I F-\alpha$

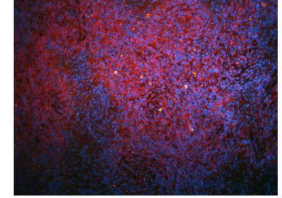

D DAPI

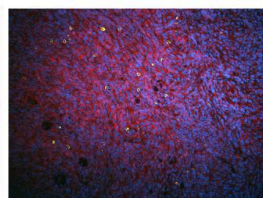

回

四

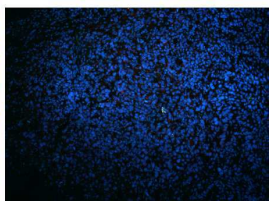

CD31

Figure S13. (a) Immunofluorescence images of HIF- $\alpha$, CA9 and CD31 of tumors after 12 days of treatment with PBS, PpIX, CCN and PCCN. 


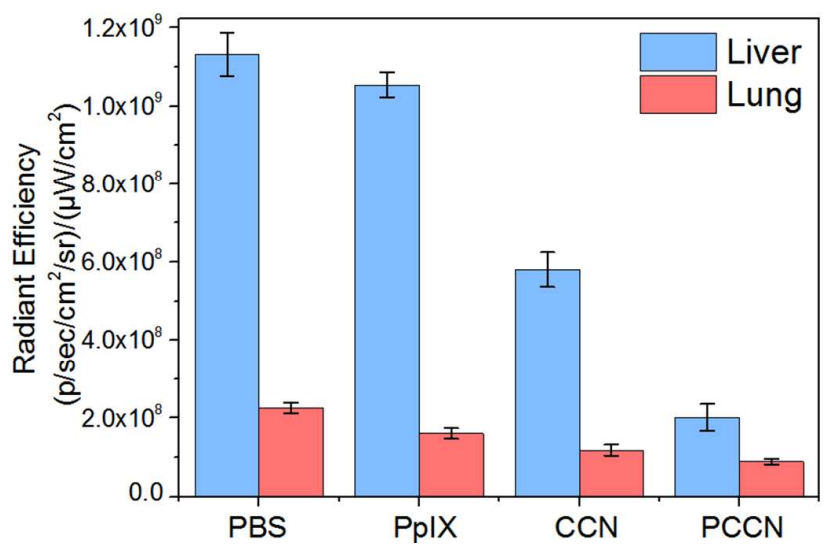

Figure S14. Quantitative analysis of lung and liver metastasis after PBS, PpIX, CCN and PCCN treatments. 